\section{From aging to cancer: a DNA methylation journey}

\author{
Natalia Puig, Ruben Agrelo \\ Epigenetics of Cancer and Aging \\ Laboratory, Institut Pasteur de \\ Montevideo, Montevideo, Uruguay
}

\begin{abstract}
Epigenetic gene silencing through DNA promoter hypermethylation is now recognized as a major step in the neoplastic transformation of the cell. The methylation levels of several genes increase with age in normal tissues such as the prostate or colon. Genes like WRN or $L M N A$ are involved in progeria, a premature aging disease. WRN and $L M N A$ are epigenetically inactivated in cancer. In both aging and cancer, global DNA methylation decreases, potentially accounting for the characteristic genomic instability of these processes. In this review, we will focus on how the accumulation of changes in DNA methylation during aging impact tumorigenesis.
\end{abstract}

\section{Introduction}

Epigenetic changes that take place during the neoplastic process in cells have been a focus of study for more than a decade. ${ }^{1}$ Moreover, epigenetic changes, including DNA methylation, acetylation, ubiquitination and phosphorylation of histone tails, could also play an important role in cellular senescence and organism aging. ${ }^{2}$

In cancer, DNA methylation of promoters at CpG islands (CGIs) plays a critical role in silencing expression of some genes, including tumor-suppressor genes. 1 There is also a decrease in global DNA methylation that may cause the genomic instability that is characteristic of the cancer cell. 1

Both an increase in DNA methylation of promoters and a decrease in global DNA methylation have been reported in aged tissues.3,4 These aging-related methylation changes may cause the well-known epigenetic changes observed in cancer, such as gene silencing, associated with CGIs promoter hypermethylation and genome instability.

Furthermore, certain genes that play a dual role in cancer and aging, such as WRN and LMNA, both of which are involved in the pathogenesis of progeria, are epigenetically silenced through the methylation of their promoters. 5,6 $W R N$ is silenced in a wide variety of tumors, while $L M N A$ is silenced in lymphoma and leukemia.5,6 Moreover, recent evidence suggests that WRN can be subject to alterations in DNA methylation in different aged tissues, as has been previously proposed.2

\section{DNA methylation: an overview}

DNA methylation is probably the best documented example of epigenetic modification. This modification involves the attachment of a covalent methyl (CH3) group to position $\mathrm{C} 5$ of the cytosine ring of Cytosine-Guanine pairs (CpGs) in the genome (5meC). ${ }^{1}$ It is estimated that, in mammals, about $60-90 \%$ of cytosines within $\mathrm{CpGs}$ are constitutively methylated with the exception of CGIs CGIs. CGIs are dense clusters of $\mathrm{CpG}$ dinucleotides, at least 200 base pairs (bp) long that are situated in or near gene promoters and often mark transcription initiation sites. In normal cells, CpG methylation plays a critical role in maintaining gene silencing. This process of gene silencing is necessary for a wide variety of cell functions, including tissue- and developmentspecific gene expression, X-chromosome silencing, genomic imprinting, and protection against expression of intragenomic parasite elements. ${ }^{1}$

\section{Methylated CpGs (mCpGs)}

In normal mammalian cells, the $\mathrm{CpG}$ dinucleotide is underrepresented due to spontaneous deamination of methylated cytosines and inaccurate repair to thymines. ${ }^{7,8}$

$\mathrm{mCpGs}$ are distributed all over the genome, either in gene bodies, intergenic regions or non-coding repetitive elements. ${ }^{9}$

More than a third of the human genome is composed of gene bodies, or the transcribed portions of genes. CpG pairs of gene bodies are methylated, as described previously, ${ }^{10}$ accounting for the majority of the methylated genome CpGs.10-12 (Figure 1A)

About $20 \%$ of all internal exons are densely methylated across their entire length, while only small regions within introns are methylated.13 The significance of this difference still needs to be clarified, ${ }^{14}$ but one possibility is that this contributes to splicing regulation. 15

Overall, it can be argued that gene-body methylation may specifically protect transcriptionally-active regions from deleterious processes, such as initiation of improper pseudo gene-body transcription, incorrect expression of the parent gene and/or chromosomal aberrations. ${ }^{16}$ Gene-body methylation may also help to regulate transcription elongation rates 16 and control alternative promoter usage 16,17 (Figure 1A). Notably, some intragenic CpGs colocalize with sites of antisense non-coding RNA ( $n c R N A)$ transcription initiation, such as Air, and Tsix, which negatively
Correspondence: Ruben Agrelo, Epigenetics of Cancer and Aging Laboratory, Institut Pasteur de Montevideo, Mataojo 2020, 11400, Montevideo, Uruguay.

Tel. +598.2.5220910 - Fax: +598.2.5224185.

E-mail: agrelo@pasteur.edu.uy

Key words: $\mathrm{CpG}$ islands, age-related methylation, tumor-suppressor genes, Werner syndrome, cancer.

Acknowledgments: we sincerely apologise to all our colleagues whose important work is not cited due to space limitations. This work was supported by Agencia Nacional de Investigacion e Inovacion (Program INNOVA URUGUAY-DCIALA/2007/19.040 URUGUAY-EUROPEAN UNION).

Received for publication: 22 September 2011. Accepted for publication: 5 November 2011.

This work is licensed under a Creative Commons Attribution NonCommercial 3.0 License (CC BYNC 3.0).

(C) Copyright N. Puig and R. Agrelo, 2012

Licensee PAGEPress srl, Italy

Ageing Research 2012; 4:e4

doi:10.4081/ar.2012.e4

regulate the expression of the sense transcript. $18-20$ HOTAIR, an $n c R N A$ transcribed from the HOXC locus, can repress in trans the expression of the $H O X D$ cluster. In all the aforementioned cases, methylation can repress the respective non-coding RNA, consequently derepressing the target gene $(\mathrm{s}) .^{21}$

Moreover, some methylated $\mathrm{CpG}$ clusters can regulate replication origin activity, as in the case of ori- $\beta$ in the dihydrofolate reductase (DHFR) locus of Chinese hamster ovary (CHO) cells. ${ }^{22,23}$

The role of intergenic hypermethylated CpGs in gene transcription is less clear. For instance, parent-specific methylation at an upstream intergenic $\mathrm{CpG}$ cluster in the H19/IGF2 imprinted locus prevents the association of the insulator element CTCF and determines expression of the imprinted locus. ${ }^{24}$

Finally, $35-50 \%$ of the human genome is composed of interspersed transposon-derived repeats, such as Long Interspersed Nuclear Elements (LINE-1) and Alu elements ${ }^{25}$ (Table 1). These interspersed repeats are often rich in $\mathrm{CpG}$ regions, which are methylated very early during embryonic development. ${ }^{25-29}$

Heterochromatic regions of telomeres (subtelomeric regions) are mostly composed of minisatellite extragenic variable number tandem repeats (minisatellite-9-64pb-VNTRs) (Table 1).4 Some VNTRs have a high content of methylated $\mathrm{CpGs}^{4}$ (Table 1). Although the role of epigenetic regulation of VNTRs still needs to be clarified, ${ }^{30}$ the involvement of macrosatellite VNTR (several kb) in certain diseases, such as 
Fragile X syndrome and Facioscapulohumeral Dystrophy (FSHD), has shed some light on this phenomenon. In Fragile X syndrome, an expansion of the promoter $\mathrm{CpGs}^{31}$ of the FMR1 gene triggers abnormal DNA methylation, which in turn leads to FMR1 silencing. ${ }^{32-34}$ On the other hand, FSHD is thought to be caused by reduced methylation of the D4Z4 macrosatellite ${ }^{35}$ due to the reduced VNTR length present in the disease. 36,37

What is more, pericentromeric regions are partly composed of methylated GC-rich Satellite II and III 4 (Table 1).

\section{CGls and $\mathrm{CpG}$ shores}

In contrast to other genome regions, CGIs are regions of permissive chromatin state and they are typically 200-3000bp in length. CGIs are defined by sequence characteristics ${ }^{1}$ because they constitute a high proportion of repetitive CpGs . CGIs surround gene promoters, Transcription Start Sites (TSSs), and/or first exons, and remain constitutively free of DNA methylation in normal cells.

In approximately $60-70 \%$ of human genes, including almost all housekeeping genes and some individual genes, promoters overlap with non-methylated CGIs for a length of $1 \mathrm{~kb} .38$

Although not all these CGIs localize to annotated TSSs, it is likely that all CGIs represent transcriptional initiation sites that have not yet been characterized. Interestingly, recent reports have found that almost $50 \%$ of replication origins are located in or close to CGIs..$^{39-41}$

In fact, it was proposed earlier that CGIs may be footprints of promoters that are associated with replication origins. ${ }^{42,43}$

Recently, a new class of intragenic CpGs has been defined: the $\mathrm{CpG}$ shores. ${ }^{44}$

CpG shores are low density $\mathrm{CpG}$ regions in close proximity (approximately $2 \mathrm{~kb}$ ) to classical CGIs that also extend beyond the flanks of the classical CGIs (Figure1). Their methylation is tightly linked with transcriptional inactivation, and it has been shown that most of the tissue-specific intragenic $\mathrm{CpG}$ methylation occurs at these CpG shores instead of at classical CGIs ${ }^{45,46}$ (Figure 1B).

\section{DNA methylation machinery and function}

DNA methylation is catalyzed by the DNA methyltransferase (DNMTs) family of enzymes, which transfer the methyl group from $\mathrm{S}$-adenosyl methionine (SAM) to the cytosine ring. 1,7

Thus far, five types of DNMTs have been identified in mammals: DNMT1, DNMT2, DNMT3a, DNMT3b and DNMT3L. Curiously, only three of the members of this family pos-

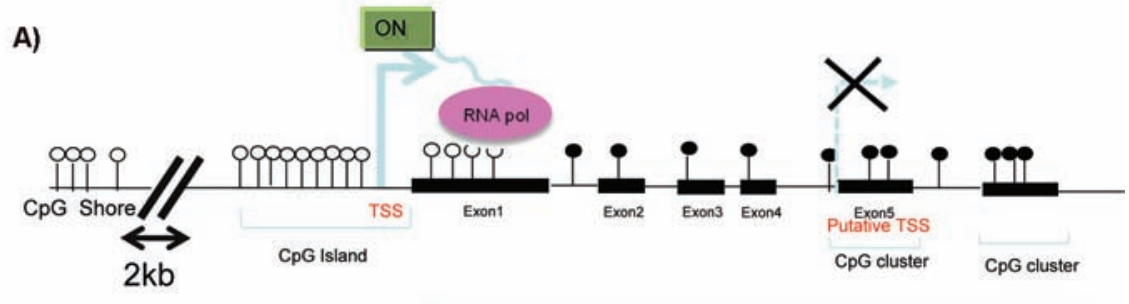

Gene body methylation (Intragenic region)

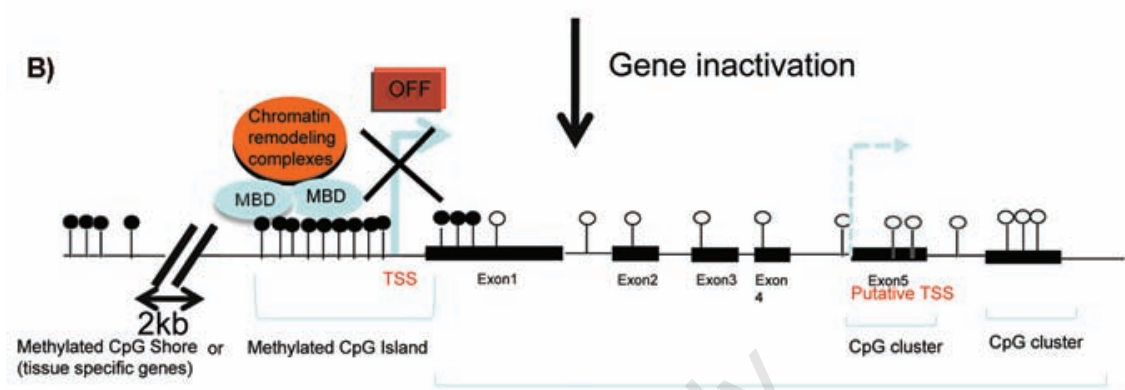

Gene body. (Intragenic region)

Figure 1. Gene inactivation due to methylation. (A) Gene representation showing genomic features, including $\mathrm{CpG}$ shores and $\mathrm{CpG}$ Islands at the promoter region and the intergenic region. A CpG shore in an unmethylated state, located about $2 \mathrm{~kb}$ from the transcription start site (TSS - sky blue arrow) is shown. A CpG island located at the promoter and part of the first exon is represented. The gene body or intragenic region is methylated. Locations where CpG clusters could coincide with putative TSS CpGs are represented as white lollipops. (B) CpG shores can become methylated and inactivate a gene that is located $2 \mathrm{~kb}$ away. This phenomenon is particularly seen in tissue-specific genes. Methylation of a $\mathrm{CpG}$ island can turn off transcription by recruiting MBD proteins and chromatin-remodeling factors. On the other hand, demethylation of intragenic $\mathrm{CpG}$ clusters could potentially activate putative TSSs.

sess methyltransferase activity: DNMT1, DNMT3a and DNMT3b.1,7 These enzymes are classified by their role in the methylation process. For example, DNMT3a and DNMT3b are de novo DNMTs and DNMT1 is a maintenance DNMT.1,7

DNA methylation patterns are established during embryogenesis by DNMT3a and DNMT3b, and are maintained throughout successive cell divisions by DNMT1. Therefore, DNMT1 catalyzes methylation patterns from a template to newly synthesized DNA strands following DNA replication 1,7 In the absence of this enzyme, the methylation patterns are gradually erased through cell divisions. This is a process referred to as passive demethylation. 14

DNMT1 is the most represented DNMT in the cell and is mainly transcribed during the $\mathrm{S}$ phase of the cell cycle. ${ }^{14}$ Even though this enzyme may exhibit de novo activity in certain circumstances, its main role in the cell is to methylate the hemimethylated sites that are produced during semi-conservative DNA replication. 14 The N-terminal domain of DNMT1 (and DNMT3a) ${ }^{47,48}$ can bind to histone deacetylases (HDACs), which promote histone deacetylation, which ultimately suppress gene expression. $^{48}$ Interestingly, gene-specific promoter methylation is abolished in cells with a mutated DNMT3b gene.14 Thus, DNMT3b may act as a transcription-coupled methyltransferase in somatic cells, in addition to its role as a de novo methyltransferase during embryogenesis. Given its limited expression level in somatic cells, ${ }^{14}$ coupling with the transcriptional machinery may focus its activity at particularly important targets. ${ }^{14}$

Despite being catalytically inactive, DNMT3L is expressed during gametogenesis and is required for the establishment of maternal genomic imprinting 49 and regulating DNMT3a and DNMT3b.50 On the other hand, DNMT2 has been reported to be responsible for tRNAAsp methylation.51 Methylation of the promoter CGIs can lead to gene suppression by at least three main mechanisms. First, it can prevent the binding of transcriptional machinery or other cis regulatory elements, such as AP252, ATF/CREB 53 , c-myc ${ }^{54}$ or $\mathrm{Sp1/Sp3,55,56}$ to their specific sequences within the promoters regions. ${ }^{57}$ Second, methylcytosine-binding proteins (MBP), such as MeCP2 and other members of the methyl-binding domain (MBD) family of proteins ${ }^{57}$ can exclude the transcriptional machinery from the promoter region. Three families of MBP have been identified; the MBD family which include MBD1-4 and $\mathrm{MeCP} 2$, the zinc finger proteis Kaiso (a 
methyl-cytosine binding protein composed of a P0Z-domain and $\mathrm{C} 2 \mathrm{H} 2$ zinc finger-domain), ZBT4, ZBTB38, and the SET-and-RING fingerassociated proteins UHRF1 and UHFR2.44 Through interactions with histone deacetylases, histone methyltransferases (e.g. HP1), and ATP-dependent chromatin remodeling enzymes, the MBDs can translate methylated DNA into a compacted chromatin environment that is repressive for transcription. ${ }^{44}$ For example, loss of MBD2 and KAISO in APC min/+ mice suppresses intestinal carcinogenesis and induces tumor-suppressor gene expression in cancer cell lines. ${ }^{58,59}$

Finally, as already mentioned, these proteins can recruit chromatin inactivation complexes, such as histone deacetylases and histone methylases, ${ }^{60}$ which lead to a condensed chromatin status at the promoter region. This results in stable transcriptional repression 7,38 and correlates with tissue-specific gene silencing, as in the case of GATA2. ${ }^{61}$

Briefly, when transcriptionally active, genes are enriched with di- and trimethylated histone H3 lysine4 (H3K4me2/me3) and have acetylation of key $\mathrm{H} 3$ and $\mathrm{H} 4$ amino acids. When genes are inactive, they lack or have minimum levels (bivalent chromatin) of these patterns but are enriched in trimethylated histone $\mathrm{H} 3$ lysines 9 and 27 (H3K9me3), and (H3K27me3) and trimethylation of $\mathrm{H} 4 \mathrm{~K} 20(\mathrm{H} 4 \mathrm{~K} 20 \mathrm{me} 3) .44$
Bivalent chromatin is mediated by the polycomb group (PcG) of proteins. PcG proteins are composed of two repressive complexes: the repressive complex 1 (PRC1) and 2 (PRC2). PRC1 contains Bmil/Mel18, MPH 1/2, Ringla/b and CBX, whereas PRC2 contains EED, Suz12 and lysine methyltransferase EZH1/2.62

While PRC2 initiates long-term repression of target genes by forming $\mathrm{H} 3 \mathrm{~K} 27 \mathrm{me} 3$, and to a lesser extent $\mathrm{H} 3 \mathrm{~K} 9 \mathrm{me} 3$, PRC1 recognizes H3K27 and maintains a silenced state by monoubiquitination of H2AK119.62 It has been suggested that PRC-mediated transcriptional silencing predisposes PcG target genes to hypermethylation of the $\mathrm{CpG}$ island. Recently, EZH2 and CBX7 have been linked to DNMTs, suggesting a role for these proteins in inducing and targeting methylation to $\mathrm{CpG}$ islands. ${ }^{63,64}$

It is well-established that CGI methylation is intimately associated with gene silencing. In normal cells, DNA methylation plays an important role in imprinting, where hypermethylation at the locus of one of the parents determines monoallelic expression. $65-67$

Moreover, DNA methylation is involved in Xchromosome inactivation (maintenance phase) in female cells. ${ }^{65}$

Tissue-specific genes can also be regulated by methylation, as is the case for genes involved in developmental processes, such as
PAX6, OSR1 and the Homeobox Ho x superfamily. $67-70$

However, further study is required to test the hypothesis that tissue-specific DNA methylation in certain genes plays a role in cell identity.

Importantly, DNA methylation in repetitive elements can protect the genome from reactivation of endoparasitic sequences.

Interestingly, several genome-wide studies have analyzed DNA replication timing and found a tight coordination between gene regulation and replication timing, in that GC-rich regions replicate early and AT-rich regions replicate late. ${ }^{41}$

\section{DNA hypomethylation: from aging to cancer}

It is well-known that advancing age is one of the major risk factors for developing tumors in humans. ${ }^{71}$ Cancer and aging are two processes that are characterized by global hypomethylation and focal hypermethylation of $\mathrm{CpG}$ islands. ${ }^{23,72}$ The term hypomethylation refers to a decrease from the normal methylation level of DNA.72

Early studies have indicated that DNA methylation patterns may be of significance in the aging process. For example, as opposed to

Table 1. Classification of silenced repeat elements during the aging and cancer processes. ${ }^{26}$

\section{Non-transposable tandem arrays of short sequences}

Macrosatellites are blocks of hundreds of $\mathrm{kb}$ with a repeat size of several kb. They are VNTRs and can be rich in $\mathrm{GC}$ content.
Satellites have sequences greater than $100 \mathrm{~kb}$ and are composed of repeats from 5 to 200bp in length. Centromeric alphoid or Satellite alpha is composed of $171 \mathrm{bp}$ sequence that is repeated many times, resulting in a region of about $500 \mathrm{~kb}$. Satellite beta (Sau3A family) is composed of a 68bp sequence that is repeated many times. Pericentromeric regions are composed of Satl (25-48bp), 2 and 3 (5bp). Sat2 and 3 are more abundant than Satl and can undergo demethylation during aging.
Mini-Satellites are members of the Telomeric family. The sequences are less than $20 \mathrm{~kb}$ long and are composed of repeats less than 25bp in length. Telomeres have tandem repeats of $6 \mathrm{bp}$ (TTAGG) that are not subject to hypomethylation.

Subtelomeric family (9-64bp): sequences are located in subtelomeric regions. They can can suffer from hypomethylation. The number of repeats varies among individuals, so they are known as variable numbers of tandem repeats, or VNTRs.
Active DNA transposons are 2-3kb in length and contain the transposase gene flanked by terminal repeat sequences. Inactive transposons have inverted repeats but lack the transposase gene. They spread using a copy-paste mechanism and represent $3 \%$ of the human genome.
LTR retrosposons or Retroposons with a long terminal repeat (LTR). Retrosposons are retrovirus-like elements and contain long terminal repeats and necessary signals to allow transposition within the genome. This process requires transcription to form a DNA copy which then integrates into the genome. They transpose via an intermediate RNA and represent $8 \%$ of the genome.

LINEs or Long interspersed elements are retroelements of $6-8 \mathrm{~kb}$ in length that do not have the LTR sequence. They encode two proteins involved in the transposition of the sequence within the genome. The mechanism used during transposition is inefficient and frequently results in truncated forms of the LINEs being transposed. They transpose via RNA intermediates and represent $20 \%$ of the genome.
Microsatellites are normally less than $150 \mathrm{bplong}$ and are composed of 1-4bp sequences. They are also VNTRs .Microsatellites rich in GC content can suffer from hypomethylation
SINEs or Short interspersed elements are short sequences of DNA about 100-300bp long. They cannot transpose without using functions provided by LINE elements. The most abundant SINE is the Alu element which has more than $1 \times 106$ copies in the genome and is $300 \mathrm{bp}$ in length. Alu elements transpose via an intermediate RNA and represent $20 \%$ of the genome. 
immortal cell lines, there is a decrease in the $5 \mathrm{meC}$ content of DNA in cultured normal diploid mouse, hamster or human fibroblasts as their number of cell divisions increases. ${ }^{73}$

Because of these findings, in 1985 Holliday proposed that the loss of $5 \mathrm{meC}$ may function as a mechanism to monitor the number of times a cell is capable of dividing during its lifetime. ${ }^{74}$ Subsequently, multiple studies established that normal aging cells derived from mice, rats and humans show a progressive loss of $5 \mathrm{meC} .75-78$

Age-related methylation was also observed in lymphocytes, in addition to fibroblasts. 79

Indeed, a developmental analysis of $5 \mathrm{meC}$ content in various mouse tissues of different ages has demonstrated that the rate of loss of methylated cytosines is inversely correlated to life span. 75

Moreover, in vitro studies of human fibroblasts (MRC-5) showed that artificially-induced DNA demethylation, with the demethylating agent 5-aza-2'-deoxycytidine (5-aza-Dc), substantially shortened the life span of the cells. ${ }^{80,81}$

Because much of the human genome (about $55 \%$ ) consists of repetitive elements, which contain the LINE-1 and Alu elements (representing approximately $30 \%$ of the total), 25 and because the majority of cytosine methylation take place within the CG-rich repetitive DNA sequences of the genome, $, 25,82,83$ it is not sur- prising that interspread transposons as well as minisatellite and tandem repeats ${ }^{84}$ become hypomethylated over time, ${ }^{85,86}$ and contribute significantly to the global genetic demethylation that comes with age (Figure 2). ${ }^{2}$

In addition to the decreased methylation status of the repetitive sequences of the genome, there is also a decrease in the methylation status and activation of numerous single-copy genes with age, ${ }^{87-90}$ such as c-myc in mouse spleen, NR2B in mouse brain, and the estrogen receptor I gene methylation in rat mammary glands. $87,88,90$

DNA hypomethylation with age has been found to reactivate imprinted genes, such as IGF2 in mouse prostatic tissue. ${ }^{91}$

A model of cellular aging led to the hypothesis that age-dependent DNA hypomethylation could affect the replication activity of origins located in CpG clusters (not associated with promoters), which are active when the clusters are methylated, reducing the DNA replication capacity of the cell. ${ }^{22,23}$ In support of this hypothesis, the demethylation of ori- $\beta$ in the DHFR locus of $\mathrm{CHO}$ cells by 5 -aza led to a reduction in its activity. 22

Cells approaching senescence exhibit decreased DNMTA-methyltransferase activity, leading to a global decrease in DNA methylation. ${ }^{92,93}$ DNMT1 has been found to be downregulated in aged cultured fibroblasts. This observation was confirmed in vitro and in vivo, proving that expression of DNMT1 and DNMT3a decreased with aging. ${ }^{92,93}$

The exact mechanism of DNA hypomethylation is still unclear, but it has been proposed that the loss of methylation may reflect the inefficiency of DNMT1 to maintain the hypermethylated status of repeated heterochromatic DNA. ${ }^{92,93}$ Consistently, a deletion of DNMT1 in mice shortens their lifespan. ${ }^{94}$ Finally, the increased activity of DNMT3b that was observed in aged cultured fibroblast ${ }^{93}$ may reflect a mechanism to compensate for the loss of methylation in repetitive DNA.2

DNA hypomethylation is a central feature of both human aged cells and cancer cells. Therefore, there are several ways in which hypomethylation can contribute to the neoplastic process. First, hypomethylation could contribute to carcinogenesis when silenced transposons (such as Line-1 and Alu) are transcribed in or relocated to other genomic regions, resulting in a disruption of the function of cellular genes. (Figure 2)

Reactivated LINE-1 transcripts may insert themselves into functional sequences, participating in homologous recombination and deregulation of protein expression, which may contribute to carcinogenesis. ${ }^{95}$

Individuals with LINE-1 hypomethylation showed increased risk of developing cancers. ${ }^{95}$

Regarding cancer prognosis, hypomethylation of LINE-1 elements in human tumor tis-

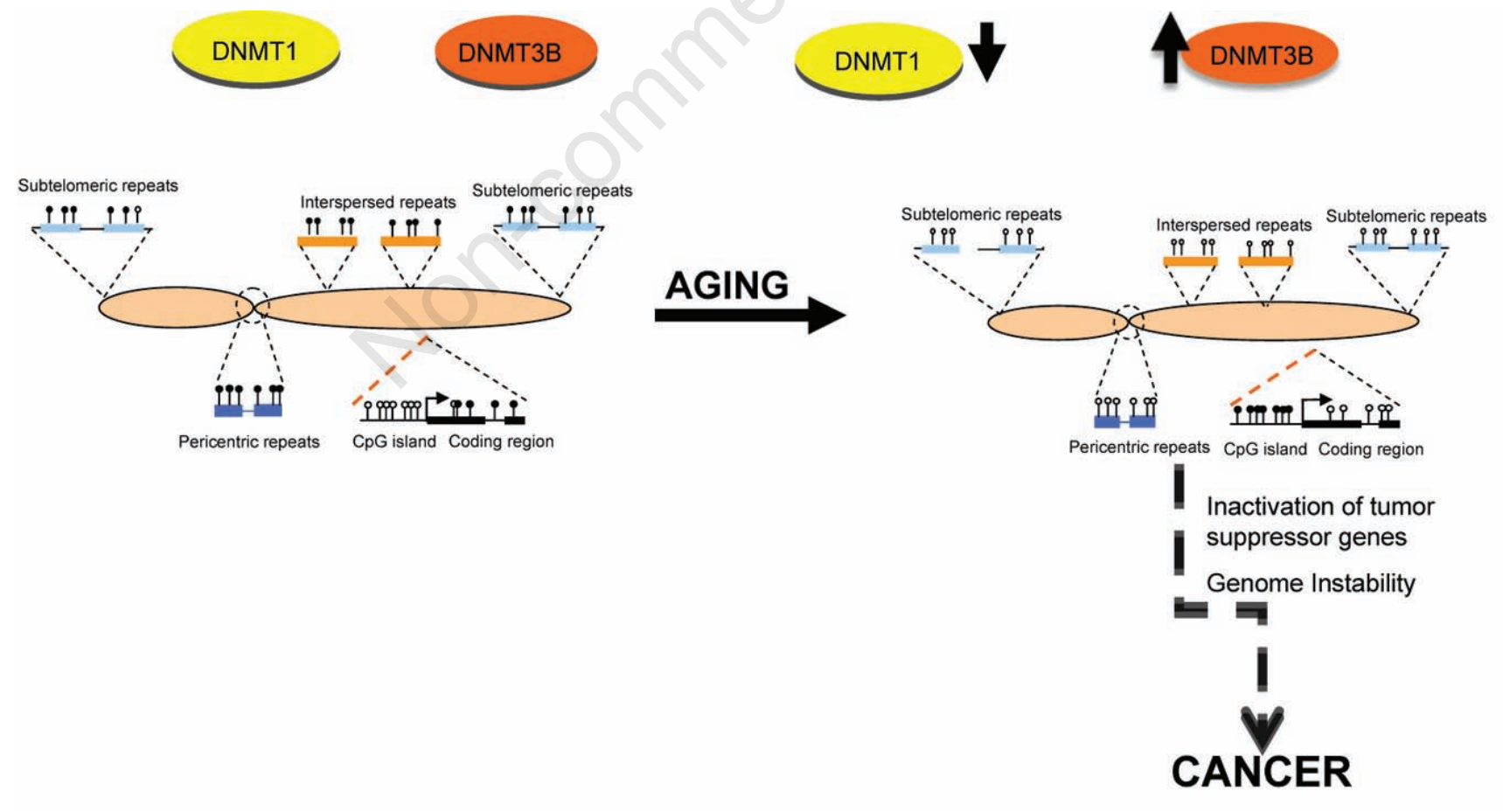

Figure 2. During the aging process, there is an increase in DNMT1 levels (yellow) that may cause demethylation of interspersed repeats (orange), subtelomeric repeats (skyblue) and pericentric repeats (blue). As a result, an increase in the DNMT3b levels (orange) may cause hypermethylation of gene promoters. These changes in the methylation patterns during the aging process can produce genome instability. This could be due to global hypomethylation or inactivation of tumor-suppressor genes by gene promoter hypermethylation, ultimately leading to cancer. 
sues or serum has been associated with poorer survival of patients with cancer ${ }^{96,97}$ and lower methylation of LINE-1 together with, lower methylation of Alu elements was shown to be associated with increased cancer mortality. 95 It has been postulated that repetitive DNA element hypomethylation can be a preceding cause for cancer occurrence. 95

Moreover, hypomethylation can trigger genomic instability by inducing genomic recombination, resulting in chromosome breaks, translocation or allelic loss ${ }^{2}$ In particular, hypomethylation at pericentromeric regions of chromosomes can cause chromosome breakage at those regions, and aneuploidy. ${ }^{2}$

For example, patients with ICF (Immunodeficiency, Centromere instability and Facial anomalies) syndrome have a significant number of chromosomal aberrations. ${ }^{98}$

This syndrome is caused by a mutation in the DNA-methyltransferase-3b (DNMT3b) gene which is associated with hypomethylation at pericentromeric regions. ${ }^{98}$

These patients also have hypomethylated subtelomeric regions, and telomeres are abnormally short in both the telomerase-positive and -negative cells ${ }^{99}$ with this phenomena probably mediated by TERRA, a long non-coding RNA. 99

These findings are in contrast to other mouse studies that showed that DNA hypomethylation in subtelomeric regions of cells lacking the enzymes responsible for DNA methylation both increased Telomere Sister Chromatid Exchange (T-SCE) and telomere elongation, independently of heterochromatic marks. 100

Second, DNA hypomethylation can cause loss of normal imprinting patterns. Indeed, studies in mice have demonstrated that this loss could itself be tumorigenic. 101 For example, IGF2 is hypomethylated in several tumor types, including breast, liver, lung and colon. 102

Third, hypomethylation at specific promoters can activate expression of oncogenes. This seems to be the case for MASPIN, which becomes hypermethylated in epithelial cells of the prostate and breast, 103 but is hypomethylated in other tumor types where its expression is correlated with the degree of dedifferentiation. ${ }^{104,105}$ Other examples include S100P in pancreatic cancer, SNCG in breast and ovarian cancer, DPP6 and oncogenic micro-RNAs. ${ }^{106}$ The melanoma-associated gene (MAGE1-A) represents an interesting case because it is located on the X-chromosome. 106

Lastly, there is substantial evidence to support the idea that global hypomethylation correlates with different stages of cancer progression and metastasis in various tumor types, including brain, prostate, hepatocellular, and cervical cancers. ${ }^{107-110}$ Furthermore, hypomethylation may appear at earlier stages of cell transformation, such as in pre-invasive colon polyps. ${ }^{111}$

\section{DNA hypermethylation: from aging to cancer}

During the last decade, great progress has been made to support the hypothesis that DNA gene promoter methylation during aging contributes to cancer. As mentioned before, the expression of DNMT3b is up-regulated in aged normal diploid fibroblasts, probably as a natural response to loss of methylation in repetitive sequences. It is possible that upregulation of DNMT3b leads to aberrant promoter hypermethylation in CpG islands that are not methylated in normal cells, although this has not been demonstrated. ${ }^{2}$

The first gene identified as becoming hypermethylated with age was the proto-oncogene cmyc in the liver of aged mice. ${ }^{112}$ It was also demonstrated that c-fos, another proto-oncogene, is progressively methylated in aged mouse and human liver. ${ }^{113}$ However, methylation of c-myc, even though there was a decrease in the transcript with age, was located distant to the promoter. This was also true for c-fos.

Importantly, a set of gene promoters were identified to be hypermethylated with age in the human liver. These included RAS association family 1 (RASSF1A), adematosis polyposis coli $(A P C), p 16$, and glutathione S-transferase p1 (GSTP1), among others.114

The gastrointestinal tract has also been intensively studied. In the duodenum, the following genes have been investigated: helicase-like transcription factor (HLTF), Pcaherin (CDH3), LIM homeobox protein 1 (LHX1), 0-6-methylguanine-DNA methyltransferase (MGMT), ubiquitin carboxyl-terminal esterase L1(UCHL1), suppression of tumourigenicity 14 (ST14), serum deprivation response factor-related gene product that binds to c-kinase (SRBC), HPP1 and SOCS1.115,116

In the duodenum, pancreas and gallbladder, it was found that methylation levels of cyclin D2 increased with age. 117

Overall, the methylation of various promoter regions of genes (hMLH1, DAP-kinase, E-cadherin, GSTP1, RASSF1A, AP, p16, RUNX3) were described in a number of organs, including the lung, kidney and liver. 118

In the human colon, the oestrogen receptor (ER) gene, 119 insulin-like growth factor II, 120 N33 and MYOD ${ }^{121}$ were methylated with age.

It has also been proposed that promoter hypermethylation of $\mathrm{CpG}$ islands in aging can change the replication timing of the replication origins located in these islands when methylated. 40

Methylation of $\mathrm{CpG}$ islands in gene promoter regions is tightly associated with aberrant silencing of transcription, and along with mutations, is one of the best-characterized mechanisms contributing to the inactivation of tumor-suppressor genes in cancer. In fact, hypermethylation affects more genes than do DNA sequence mutations. ${ }^{122}$ Epigenetic silencing could inactivate one or both alleles of a gene or could coexist with a mutation or deletion in the opposite allele. ${ }^{23}$ Epigenetic silencing of specific genes can affect virtually all of the mechanisms and stages of cancer development. Some of these include, among others, the DNA repair pathway ( $h M L H 1, M G M T$, WRN, BRCA1), Ras signaling (RASSFIA, NOR$E I A)$, the cell cycle $(p 16, p 15, R B)$ and the p53 pathway (p14, p73, HIC1). ${ }^{1}$

Hypermethylation patterns have been found to be tumor type-specific, but it is still unknown why some regions become hypermethylated. Current studies suggest that hypermethylation in cancer is the result of non-specific de novo methylation of particular genes that confers some advantage to the cancer cells, resulting in clonal selection. The second possibility is that highly methylated sequences can be methylation seeds that spread to the surrounding genomic regions. Interestingly, it has also been suggested that CGIs promoter hypermethylation is pre-programmed by $\mathrm{PcG}$ that are normally involved in the regulation of embryonic genes. ${ }^{124}$

Very recently, genomic architecture has been found to be an important factor in promoting CpG island methylation. In the reported study, a remote location from SINE and LINE was found to be associated with the susceptibility of promoter CGIs to aberrant hypermethylation during carcinogenesis. This was independent of epigenetic factors such as RNA poll and the repressive histone mark H3K27me3.125 Moreover, a link between high order chromatin folding PcG repression and CGIs methylation has been established in colorectal cancer. One study found PcG/H3K27me3 enriched elements at the base of a chromatin loop intersection point over a $100 \mathrm{~kb}$ region at a GATA4 non-transcribed locus, but this structure was not present in cells that expressed GATA4.126 These data support the idea that PcG is involved in determining a three-dimensional repressive chromatin structure. 126

Interestingly, transcription requires the removal of nucleosomes fromTSS. Therefore, promoter hypermethylation results in the occupation of the transcription start site by a nucleosome, as has been described for $h M L H 1$ in colon cancer. ${ }^{127}, 128$

Recently, a phenomenon named Long Range Epigenetic Silencing (LRES) was described. Epigenetic silencing can span large chromosomal regions that contain a significant number of genes. Several tumor-suppressor genes can be methylated simultaneously. ${ }^{129}$ For example, LRES has been reported for a 4/MB region on chromosome 3p22 that includes the MLH1 gene in Microsatellite Instability Colorrectal Cancers (MSI-H-CRC). 130 
It is also well-known that in certain subsets of cancers, multiple tumor-suppressor genes could be systematically disrupted.

This alteration is known as $\mathrm{CpG}$ island methylator phenotype (CIMP), and in a subset of colon cancers, this has been associated with a genotype that includes mutations of the $B R A F$ oncogene and microsatellite instability $(M S I)$ due to promoter hypermethylation of hMLH1.131

To conclude, it is important to remember that some genes, such as Ras association gene (RASSF1A), p14, p16 and hMLH1, are suppressed by hypermethylation in cancer and are also subject to age-related methylation, linking both processes (Figure 3).

\section{WRN and LMNA: epigenetically silenced progeroid genes}

Because aging is the main biological phenomenon associated with the risk of developing cancer, it is not surprising to find that genes which are involved in safeguarding the genome against aging are inactivated in cancer cells.

The first example is the epigenetic silencing of the $W R N$ gene. The mutation of this gene causes adult progeria, or Werner syndrome (WS).

WS is a very rare autosomal recessive human disorder that is characterized by the appearance of premature aging. 132,133 WS patients often suffer from type 2 diabetes, arteriosclerosis, cataracts, and osteoporosis. They also exhibit a short stature, premature graying and loss of hair, skin problems such as scleroderma, characteristic pitched voice and regional subcutaneous fat atrophy. The two main causes of death in WS patients are arteriosclerosis (44\%) and neoplasia (39.5\%). ${ }^{133,134}$ Moreover, the type of neoplasms in WS patients is not the same as in cancer patients without this syndrome; the ratio of mesenchymal to epithelial cancers is 1:1 in WS patients 134,135 and 1:10 in the normally aged population. However, almost all types of tumors are found in WS patients. $136 \mathrm{WS}$ cells show increased chromosomal instability, with deletions, reciprocal translocations and inversions, in what is known as variegated translocated mosaicism. ${ }^{137}$

$W R N$ is a member of the RecQ helicase family. This family includes the genes RecQ, RecQ5, and RecQ4, which is associated with Rothmund Thompson syndrome, and BLM, which is associated with Bloom syndrome. WRN possesses DNA-dependent ATP-ase activity and 3'-5' helicase and exonuclease activity. $138-140$

WRN participates in diverse pathways, including repair, replication, telomere metabo-

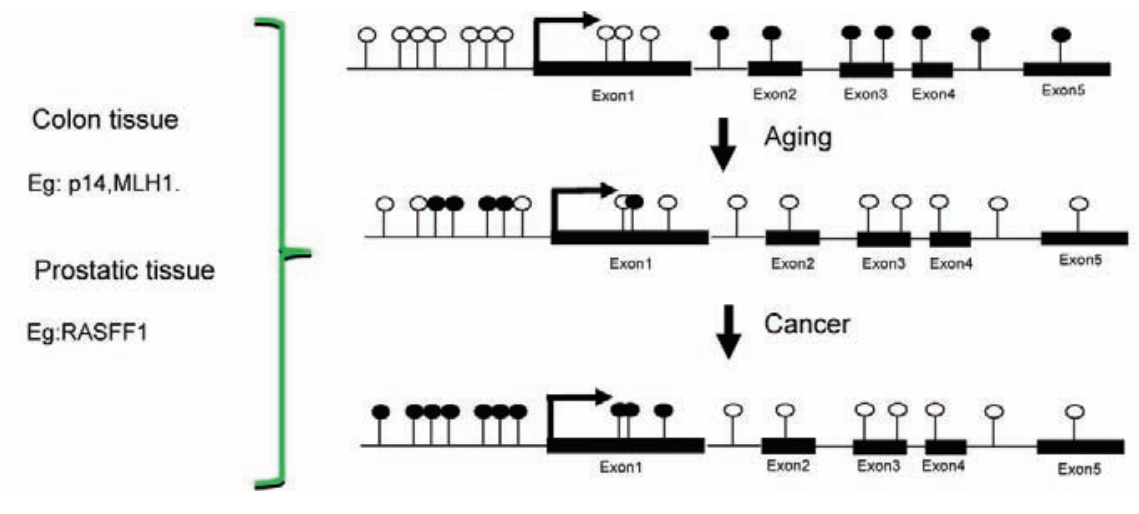

Figure 3. Genes involved in DNA repair and tumor-suppressor genes can be inactivated by gene promoter hypermethylation in the aged tissue, and this suppression can contribute to neoplastic transformation of the cell. For example, this could happen in colon tissue or prostatic tissue.

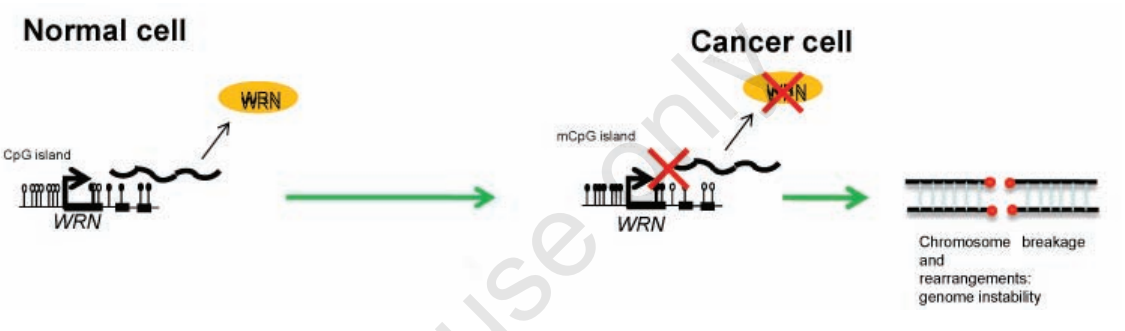

Figure 4. WRN can be epigenetically silenced in a wide variety of human cancers, contributing to cell transformation due to genome instability. WRN is involved in several pathways of DNA repair, and its absence could produce chromosome breaks. WRN could be considered a type of caretaker of tumor-suppressor gene.

lism, and p53-mediated pathways. ${ }^{139,140}$

WRN has been found to be inactivated by CpG island promoter hypermethylation in a wide variety of tumors of mesenchymal and epithelial origin. 5

The re-introduction of the $W R N$ gene into tumor cells with the epigenetically inactivated $W R N$ produces tumor-suppressor-like features. Moreover, when tumor cells whose WRN promoter had been silenced by hypermethylation were treated with 5-AZA, the exonuclease activity of the $W R N$ protein was restored. 5 Notably, upon exposure to DNA-damaging agents, cancer cells with $W R N$ promoter hypermethylation and cells from WS patients were highly sensitive to these drugs, with a high frequency of chromomosomal breakage, in striking contrast to cancer cells with unmethylated $W R N$ promoters. This sensitivity was abolished in $W R N$-methylated cells, upon transfection of WRN (Figure 4). 5

Interestingly, significant age-related methylation alterations in $W R N$ in different tissues were recently found. 141

The second example of this type of gene is LMNA.

LMNA-type proteins, together with B-type lamins and lamin-associated proteins, are components of the nuclear lamina. ${ }^{142,143}$

The nuclear lamina is a network of fila- ments and proteins that is located on the inner side of the nuclear membrane. Nuclear lamins are extremely dynamic, suggesting their role in the non-random positioning of subchromosome domains. 142,143

They are also involved in cell cycle regulation, DNA replication, differentiation, and apoptosis. ${ }^{142,143}$

Lamins can be classified into types B and A. Type B are essential for cell viability and expressed in all cell types, while type A are expressed in most differentiated somatic cells. ${ }^{142,143}$

The lamin $\mathrm{A} / \mathrm{C}$ gene encodes lamins $\mathrm{A}$ and $\mathrm{C}$, which are two isoforms that arise as a consequence of alternative RNA splicing. Mutations in human $L M N A$ cause a plethora of pathologies called laminopathies. These include atypical WS and Hutchinson Guilford Progeria (HGPS). ${ }^{143}$

$\mathrm{CpG}$ island hypermethylation of the lamin $\mathrm{A} / \mathrm{C}$ gene promoter has been found in leukemias and lymphomas, and is a predictor of poor outcome in these patients. This is particularly true for diffuse large B-cell lymphomas (DLBCL). ${ }^{6}$

Because lamin $\mathrm{A} / \mathrm{C}$ is involved in the chromatin reorganization and reprogramming necessary for differentiation, one possibility is that blocking it can facilitate an undifferentiat- 
ed phenotype that could be advantageous for tumor progression. 144

The second possibility is that its absence could destabilize RB protein, promoting cell cycle progression through E2F1, as is the case for $L M N A$-/- embryonic mouse fibroblasts (MEFS). ${ }^{145}$

However, both possibilities are not mutually exclusive.

\section{Concluding remarks}

We provide an overview of DNA methylation and its associated machinery to explore the role of methylation in the aging process and neoplastic transformation, and to demonstrate how both these processes are linked. In particular, we have focused on what is known about the global loss of DNA methylation in relation to DNMT1 downregulation in aging cells, and the consequences for cancer development. ${ }^{92,93}$ Genes that are methylated during aging, probably as a consequence of DNMT3b upregulation, are hypermethylated in cancer, which favors neoplastic transformation. ${ }^{2}$ Importantly, $W R N$ and $L M N A$, two genes involved in the aging process with roles in human progeria and tumor suppression, were found to be hypermethylated in human neoplasia. 5,6

Current technological development has reached a point where whole genome methylation scans are now possible, and these are starting to shed light on the intricacies of DNA methylation phenomena. However, one caveat of these studies in relation to aging epigenomes is that changes in aging are sometimes subtle and less frequent than in cancer epigenomes. It should be noted that, together with methylome changes, the role for transcriptional networks in controlling these changes should be studied.

Several large scale studies have revealed in both human tissue 146 and mouse intestinal tissue ${ }^{147}$ that methylation is associated with promoters of cancer-relevant genes which are methylated during the aging process. It has been suggested that age-dependent DNA methylation changes begin before adulthood ${ }^{148}$ and that different patterns of epigenetic dysregulation occur within each tissue over time. 148

Moreover, genes methylated during aging can be polycomb targets that are suppressed in stem cells. ${ }^{150,151}$

A recent observation using cultured Mesenchymal Stromal Cells (MSC), which are precursors for mesodermal cell lineages, found a concordance between methylation changes upon long-term cell cultured samples, aging samples and ovarian cancer samples.152,153 Following these results, it has been suggested that changes in the methylation patterns observed in the aging and replicative senescence process could represent a developmental program. This is opposed to stochastic events, as we discussed earlier in this review. ${ }^{148}$ However, it is more likely that both stochastic events and a developmental program could coexist during aging to some extent.

Using large-scale approaches, it has also been demonstrated that loci in $\mathrm{CpG}$ island gained methylation with age, while loci at non$\mathrm{CpG}$ islands lost methylation with age. As previously mentioned, $W R N$ has been found to have altered methylation in different tissues according to age. ${ }^{141}$

To conclude, it should be possible in the near future to address several unanswered questions. i) it will be necessary to dissect the mechanism that targets and initiates DNA methylation, as well as the mechanism that causes loss of global methylation in aging and cancer; ii) the recent discovery of $\mathrm{CpG}$ shores makes them an important target for the study of methylation in aging; iii) although the contribution of hypermethylation to the suppression of non-coding RNAs in cancer has been demonstrated, ${ }^{1}$ it will be interesting to evaluate its role in the aging process; iv) we need a better understanding of how and in what cell context PcG complexes pre-program CGIs for methylation; v) it is of fundamental importance to improve our understanding of the role nuclear structure may play in senescent cells, as well as its interplay with epigenetic markers $; 6,154 \mathrm{vi}$ ) it is important to determine if the epigenetic regulation of DNA replication origins has an important role in the aging process, as previously suggested in a model of cellular aging. 23

Tackling these questions will lead to a better understanding of DNA methylation in aging and cancer.

\section{References}

1. Esteller M. Epigenetics in cancer. N Engl J Med 2008;358:1148-59.

2. Fraga MF, Agrelo R, Esteller M. Cross-talk between aging and cancer: the epigenetic language. Ann N Y Acad Sci 2007;1100: 60-74.

3. Issa JP. CpG-island methylation in aging and cancer. Curr Top Microbiol Immunol 2000; 249:101-18.

4. Kim J, Kim Y-J, Issa JP. Aging and DNA methylation. Curr Chem Biol 2009;3:3219.

5. Agrelo R, Cheng WH, Setien F, et al. Epigenetic inactivation of the premature aging Werner syndrome gene in human cancer. Proc Natl Acad Sci USA 2006;103:8822-7.

6. Agrelo R, Setien F, Espada J, et al.
Inactivation of the lamin $\mathrm{A} / \mathrm{C}$ gene by $\mathrm{CpG}$ island promoter hypermethylation in hematologic malignancies, and its association with poor survival in nodal diffuse large B-cell lymphoma. J Clin Oncol 2005;23:3940-7.

7. Bird A. DNA methylation patterns and epigenetic memory. Genes Dev 2002; 16:6-21.

8. Mazin AL. Suicidal function of DNA methylation in age-related genome disintegration. Aging Res Rev 2009;8:314-27.

9. Klose RJ, Bird AP. Genomic DNA methylation: the mark and its mediators. Trends Biochem Sci 2006;31:89-97.

10. Illingworth R, Kerr A, Desousa D, et al. A novel $\mathrm{CpG}$ island set identifies tissue-specific methylation at developmental gene loci. PLoS Biol 2008;6:e22.

11. Rauch TA, Wu X, Zhong X, et al. A human $B$ cell methylome at 100-base pair resolution. Proc Natl Acad Sci USA 2009; 106:671-8.

12. F, Lewin J, Cortese R, et al. DNA methylation profiling of human chromosomes 6 , 20 and 22. Nat Genet 2006;38:1378-85.

13. Fabienne B, Moh M, Funk P, et al. DNA Methylation of the First Exon Is Tightly Linked to Transcriptional Silencing. PLoS ONE 2011;6:e14524.

14. Aran D, Toperoff G, Rosenberg M, et al. Replication timing-related and gene bodyspecific methylation of active human genes. Hum Mol Genet 2011; 20:670-80.

15. Estécio MR, Issa JP. Dissecting DNA hypermethylation in cancer. FEBS letters 2011;13:2078-86.

16. Mataldi NN, Denis H, Fuks F. Exposing the DNA methylome iceberg. Trends Biochem Sci 2011;7:381-7.

17. Maunakea AK, Nagarajan RP, Bilenky M. Conserved role of intragenic DNA methylation in regulating alternative promoters. Nature 2010;466:253-7.

18. Panning B, Jaenisch R. DNA hypomethylation can activate Xist expression and silence X-linked genes. Genes Dev 1996;10:1991-2002.

19. Wutz A, Smrzka OW, Schweifer N, et al. Imprinted expression of the Igf2r gene depends on an intronic CpG island. Nature 1997;389:745-9.

20. Sleutels F, Zwart R, Barlow DP. The noncoding Air RNA is required for silencing autosomal imprinted genes. Nature 2002;415:810-3.

21. Rinn JL, Kertesz M, Wang JK et al. Functional demarcation of active and silent chromatin domains in human HOX loci by noncoding RNAs. Cell 2007; 129:1311-23.

22. Rein T, Zorbas H, De Pamphilis ML. Active mammalian replication origins are associated with a high density cluster of mCpG dinucleotides. Mol Cell Biol 
1997;17:416-23.

23. Agrelo R. A new molecular model of cellular aging based on Werner syndrome. Med Hypotheses 2007;68:770-80.

24. Edwards CA, Ferguson-Smith AC. Mechanisms regulating imprinted genes in clusters. Curr Opin Cell Biol 2007;19:281-9.

25. Yoder JA, Walsh CP, Bestor TH. Cytosine methylation and the ecology of intragenomic parasites. Trends Genet 1997;13: $335-40$.

26. Strachan T, Read AP. Human Molecular Genetics. 2nd edition, John Wiley \& Sons, Singapore, 1999.

27. Monk M, Boubelik M, Lehnert S. Temporal and regional changes in DNA methylation in the embryonic, extraembryonic and germ cell lineages during mouse embryo development. Develop-ment 1987;99:37182.

28. Clark SJ, Melki J. DNA methylation and gene silencing in cancer: which is the guilty party? Oncogene 2002;21:5380-7.

29. Rubin CM, VandeVoort CA, Teplitz Rl, et al. Alu repeated DNAs are differentially methylated in primate germ cells. Nucleic Acids Res 1994;22:5121-7.

30. van Deutekom JC, Wijmenga C, van Tienhoven EA, et al. FSHD associated DNA rearrangements are due to deletions of integral copies of a $3.2 \mathrm{~kb}$ tandemly repeated unit. Hum Mol Genet 1993;2:2037-42.

31. Fu YH, Kuhl DP, Pizzuti A, et al. Variation of the CGG repeat at the fragile $\mathrm{X}$ site results in genetic instability: resolution of the Sherman paradox. Cell 1991; 67:104758.

32. Hornstra IK, Nelson DL, Warren ST, et al. High resolution methylation analysis of the FMR1 gene trinucleotide repeat region in fragile $\mathrm{X}$ syndrome. Hum Mol Genet 1993;2:1659-65.

33. Pieretti M, Zhang FP, Fu YH, et al. Absence of expression of the FMR-1 gene in fragile X syndrome. Cell 1991;66:81722.

34. Sutcliffe JS, Nelson DL, Zhang F, et al. DNA methylation re- presses FMR-1 transcription in fragile $\mathrm{X}$ syndrome. Hum Mol Genet 1992;1:397-400.

35. de Greef JC, Wohlgemuth M, Chan OA et al. Hypomethylation is restricted to the D4Z4 repeat array in phenotypic FSHD. Neurology 2007;69:1018-26.

36. Gabellini D, Green MR, Tupler R. Inappropriate gene activation in FSHD: a repressor complex binds a chromosomal repeat deleted in dystrophic muscle. Cell 2002;110:339-48.

37. van Deutekom JC, Wijmenga C, van Tienhoven EA, et al. FSHD associated DNA rearrangements are due to deletions of integral copies of a $3.2 \mathrm{~kb}$ tandemly repeated unit. Hum Mol Genet 1993;2:2037-42.

38. Weber M, Hellmann I, Stadler MB, et al. Distribution, silencing potential and evolutionary impact of promoter DNA methylation in the human genome. Nat Genet 2007;39:457-66.

39. Cadoret JC, Meisch F, Hassan-Zadeh V, et al. Genome-wide studies highlight indirect links between human replication origins and gene regulation. 2008; 15837-42.

40. Prioleau MN. CpG islands: starting blocks for replication and transcription. PLoS Genet.2009;5:e1000454.

41. Necsulea A, Guillet C, Cadoret JC, et al The relationship between DNA replication and human genome organization. Mol Biol Evol. 2009;26:729-41.

42. Antequera F. Structure, function and evolution of $\mathrm{CpG}$ island promoters. Cell Mol. Life Sci. 2003;60:1647-58.

43. Antequera F, Bird A. CpG islands as genomic footprints of promoters that are associated with replication origins. Curr Biol 1999;9:R661-7.

44. Portela A, Esteller M. Epigenetic modifications and human disease. Nature Biotechnology 2010;10:1057-68.

45. Doi A. Park IH, Wen B, et al. Differential methylation of tissue- and cancer-specific CpG island shores distinguishes human induced pluripotent stem cells, embryonic stem cells and fibroblasts. Nat Genet 2009;41:1350-3.

46. Ji H, Ehrlich LI, Seita J, et al. Com-prehensive methylome map of lineage commitment from haematopoietic progenitors. Nature 2010;467:338-42.

47. Fuks F, Burgers WA, Godin N, et al. Dnmt3a binds deacetylases and is recruited by a sequence- specific repressor to silence transcription. EMBO J 2001;20:2536-44.

48. Rountree MR, Bachman KE, Baylin SB. DNMT1 binds HDAC2 and a new corepressor, DMAP1, to form a complex at replication foci. Nat Genet 2000;25:26977.

49. Bourchis D, Xu GL, Lin CS, et al. Dnmt3L and the establishment of maternal genomic imprints. Science 2001;294: 2536-9.

50. Gowher H, Liebert K, Hermann A, et al. Mechanism of stimulation of catalytic activity of Dnmt3A and Dnmt3B DNA(cytosine-C5) methyltransferases by Dnmt3L. J Biol Chem 2005;280:13341-8.

51. Goll MG, Kirpekar F, Maggert KA, et al. Methylation of tRNAAsp by the DNA methyltransferase homolog Dnmt2. Science 2006;311:395-8.

52. Comb M, Goodman HM. CpG methylation inhibits proenkephalin gene expression and binding of the transcription factor AP-
2. Nucleic Acids Res 1990;13:3975-82.

53. Iguchi-Ariga SM, Schaffner W. CpG methylation of the cAMP-responsive enhancer/promoter sequence TGACGTCA abolishes specific factor binding as well as transcriptional activation. Genes Dev 1989;3:612-9.

54. Prendergast GC, Ziff EB. Methylation-sensitive sequence-specific DNA binding by the c-Myc basic region. Science 1991;251:186-9.

55. Clark SJ, Harrison J, Molloy PL. Sp1 binding is inhibited by (m.) $\mathrm{Cp}(\mathrm{m}$.) $\mathrm{CpG}$ methylation. Gene 1997;195:67-71.

56. Zhu WG, Srinivasan K, Dai Z, et al.Methylation of adjacent CpG sites affects Sp1/Sp3 binding and activity in the p21(Cip1) promoter. Mol Cell Biol. 2003;23:4056-65.

57. Bird AP, Wolffe AP. Methylation-induced repression--belts, braces, and chromatin. Cell 1999;99:451-4

58. Samsonm OJ, Berger J, Bishop SM, et al. Deficiency in MBD2 suppresses intestinal tumorigenesis. Nat Genet 2003;34: 145-7.

59. Lopes EC, Valls E, Figueroa ME,et al. Kaiso contributes to DNA methylation dependent silencing of tumor suppressor genes in colon cancer cell lines. Cancer Res 2008;68:7258-63.

60. Jones PA, Baylin SB. The fundamental role of epigenetic events in cancer. Nat Rev Genet 2002;3:415-28.

61. Song F, Smith JF, Kimura MT, et al. Association of tissue-specific differentially methylated regions (TDMs. with differential gene expression. Proc Natl Acad Sci USA 2005;102:3336-41.

62. Sparmann A, van Lohuizen M. Polycomb silencers control cell fate,development and cáncer. Nat Rev Cancer 2006;6:84656.

63. Mohammad HP, Cai Y, McGarvey KM, et al. Polycomb CBX7 promotes initiation of heritable repression of genes frequently silenced with cancer-specific DNA hypermethylation. Cancer Res 2009;69:6322-30.

64. Vire E, Brenner C, Deplus R, et al. The polycomb group protein EZH2 directly controls DNA methylation. Nature 2006;439:871-4.

65. Agrelo R, Wutz A. Context of change-X inactivation and disease. EMBO Mol Med 2010;2:6-15.

66. Kacem S, Feil M. Chromatin mechanisms in genomic imprinting.Mamm Genome 2009;20.544-56.

67. Kleinjan DA, Seawright A, Childs AJ, et al. Conserved elements in Pax6 intron 7 involved in (auto)regulation and alternative transcription. Dev Biol 2004;265: 462 77.

68. Illingworth R, Kerr A, Desousa D, et al. A novel $\mathrm{CpG}$ island set identifies tissue-spe- 
cific methylation at developmental gene loci. PLoS Biol 2008;6:e22.

69. Rauch TA, Wu X, Zhong X, et al. A human B cell methylome at 100-base pair resolution. Proc. Natl. Acad Sci USA 2009;106: 671-8.

70. Irizarry RA, Ladd-Acosta C, Wen B, et al. The human colon cancer methylome shows similar hypo and hypermethylation at conserved tissue-specific $\mathrm{CpG}$ island shores. Nat. Genet 2009;41:178-6.

71. De Pinho RA. The age of cancer. Nature. 2000;408:248-54.

72. Agrelo R. Epigenetic Silencing of Progeroid Syndromes. In Epigenetics of Aging. Tollefsbol, T0. ed.1sted. Springer Verlag, New York, 2010.

73. Wilson VL, Jones PA. DNA methylation decreases in aging but not in immortal cells. Science 1983;220:1055-7.

74. Holliday R. The inheritance of epigenetic defects. Science 1987;238:163-70

75. Wilson VL, Smith RA, Ma S, et al. Genomic 5-methyldeoxycytidine decreases with age. J Biol. Chem 1987 262:9948-51.

76. Singhal RP, Mays-Hoopes LL, Eichhorn GL. DNA methylation in aging of mice. Mech Ageing Dev 1987;41:199-210.

77. Richardson BC. Role of DNA methylation in the regulation of cell function:autoimmunity,aging and cancer. $\mathrm{J}$ Nutr 2002;232:2401S-05S.

78. OnoT, Uehara Y,Kurishita A, et al. Biological significance of DNA methylation in the ageing process. Age Ageing 1993;22:S34-S43.

79. Drinkwater RD, Blake TJ, Morley AA, et al. Human lymphocytes aged in vivo have reduced levels of methylation in transcriptionally active and inactive DNA. Mutat Res 1989;219:29-37.

80. Fairweather DS, Fox M, Margison GP. The in vitro lifespan of MRC-5 cells is shortened by 5 -azacytidine-induced demethylation. Exp Cell Res 1987;168: 153-9.

81. Holliday R. Strong effects of 5'-azacytidine on the in vitro lifespan of human diploid fibroblasts. Exp Cell Res 1986; 166:543-52.

82. Gama-Sosa MA, Wang RY, Kuo KC, et al. The 5-methylcitosine content of highly repeated sequences in human DNA. Nucleic Acids Res 1983;11:3087-95.

83. Rollins RA, Haghighi F, Edwards JR, et al. Large-scale structure of genomic methylation patterns. Genome Res 2006;16: 15763 .

84. Suzuki T, Fujii M, Ayusawa D. Demethylation of classical satellite 2 and 3 DNA with chromosomal instability in senescent human fibroblasts. Exp Gerontol 2002;37:1005-14.

85. Fraga MF, Ballestar E, Paz MF, et al. Epigenetic differences arise during the lifetime of monozygotic twins. Proc Nat Acad Sci USA 2005;102:10604-9.

86. Rodríguez J, Vives L, Jorda M, et al. Genome-wide tracking of unmethylated DNA Alu repeats in normal and cancer cells. Nucleic Acids Res 2008;36:770-84.

87. Ono T, Takahashi N, Okada S. Age-associated changes in DNA methylation and mRNA level of the c-myc gene in spleen and liver of mice. Mutat Res 1989;219:3950 .

88. Yenbutr P, Hilakivi-Clarke L, Passaniti A. Hypomethylation of an exon I estrogen receptor $\mathrm{CpG}$ island in spontaneous and carcinogen-induced mammary tumorigenesis in the rat. Mech. Ageing Dev 1998;106:93-102.

89. Zhang Z, Deng C, Lu Q, et al. Age-dependent DNA methylation changes in the ITGAL (CD11a) promoter. Mech, Ageing Dev. 2002;123:1257-68.

90. Ravindran CR, Ticku MK. Methylation of NMDA receptor NR2B gene as function of age in the mouse brain. Neurosci Lett 2005;380:223-8.

91. Fu VX, Dobosy JR, Desotelle JA, et al, Aging and cancer-related loss of insulinlike growth factor 2 imprinting in the mouse and human prostate. Cancer Res 2008:68:6797-802.

92. Lopatina N, Haskell JF, Andrews LG, et al. Differential maintenance and de novo methylating activity by three DNA methyltransferases in aging and immortalized fibroblasts. J Cell Biochem 2002; 84:324-34.

93. Casillas MA Jr, Lopatina N, Andrews LG, et al. Transcriptional control of the DNA methyltrans-ferases is altered in aging and neoplastically-transformed human fibroblasts. Mol Cell Biochem 2003;252: 33-43.

94. Ray D, Wu A, Wilkinson JE, et al. Aging in heterozygous Dnmt1-deficient mice: effects on survival, the DNA methylation genes, and the development of amyloidosis. J Gerontol A Biol Sci Med Sci 2006; 61:115-24

95. Zhu Z, Sparrow D, Hou L, et al. Repetitive element hypomethylation in blood leukocyte DNA and cancer incidence, prevalence, and mortality in elderly individuals: the Normative Aging Study. CCC 2011; 22:437-47.

96. Furniss CS, Marsit CJ, Houseman EA, et al. Line region hypomethylation is associated with lifestyle and differs by human papillomavirus status in head and neck squamous cell carcinomas. Cancer Epidemiol Biomarkers Prev 2008;17: 966-71.

97. Tangkijvanich P, Hourpai N, Ratta-natanyong $\mathrm{P}$, et al. Serum LINE-1 hypo-methylation as a potential prognostic marker for hepatocellular carcinoma. Clin Chim Acta 2007;379:127-133.

98. Ehrlich M, Sanchez C, Shao C, et al. ICF an immunodeficiency syndrome: DNA methyltransferase 3B involvement, chromosome anomalies, and gene dysregulation. Autoimmunity 2008;41:253-71

99. Yehezkel S, Segev Y, et al. Hypomethylation of subtelomeric regions in ICF syndrome is associated with abnormally short telomeres and enhanced transcription from telomeric regions Hum Mol Genet 2008;17:2776-89.

100. Gonzalo S, Jaco I, Fraga MF, et al. DNA methyltransferases control telomere length and telomere recombination in mammalian cells. Nature Genetics 2006;8:416-24.

101. Sakatani T, Kaneda A, Iacobuzio-Donahue CA. Loss of imprinting of Igf2 alters intestinal maturation and tumorigenesis in mice. Science 2005;307:1976-8.

102. Ito, Y, et al. Somatically acquired hypomethylation of IGF2 in breast and colorectal cancer.2008 Hum Mol Genet 2008; 17:2633-43.

103. Futscher BW, et al. Aberrant methylation of the maspin promoter is an early event in human breast cancer. Neoplasia 2004;6:380-9.

104. Futscher BW, et al. Role for DNA methylation in the control of cell type specific maspin expression. Nat Genet 2002;31: 175-9.

105. Bettstetter $M$, et al. Elevated nuclear maspin expression is associated with microsatellite instability and high tumour grade in colorectal cancer. J Pathol 2005;205:606-14.

106. Wilson AS, Power BE Molloy, PL. DNA hypomethylation and human diseases. Biochim Biophys Acta 2007;1775:138-62.

107. Cadieux B, Ching TT, VandenBerg SR, et al. Genome-wide hypomethylation in human glioblastomas associated with specific copy number alteration, methylenetetrahy drofolate reductase allele status, and increased proliferation. Cancer Res 2006;66:8469-76.

108. Kim YI, Giuliano A, Hatch KD, et al. Global DNA hypomethylation increases progressively in cervical dysplasia and carcinoma. Cancer 1994;74:893-9.

109. Lin CH, Hsieh SY, Sheen IS, et al. Genome-wide hypomethylation in hepatocellular carcinogenesis. Cancer Res 2001;61:4238-43.

110. Bedford MT, van Helden PD. Hypome-thylation of DNA in pathological conditions of the human prostate. Cancer Res 1987;47:5274-6.

111. Goelz SE, Vogelstein B, Hamilton SR, et al. Hypomethylation of DNA from benign and malignant human colon neoplasms. 
Science 1985;228:187-90.

112. Ono T, Tawa R, Shinya K, et al. Methylation of the c-myc gene changes during aging process of mice. Biochem Biophys Res Commun 1986 139:1299-304.

113. Choi EK, Uyeno S, Nishida N, et al. Alterations of c-fos gene methylation in the processes of aging and tumorigenesis in human liver.Mutat Res 1996;54: 123-8.

114. Nishida N, Nagasaka T, Nishimura T, et al.Aberrant methylation of multiple tumor suppressor genes in aging liver, chronic hepatitis, and hepatocellular carcinoma. Hepatology 2008;47:908-18.

115. Ahuja N, Issa JP. Aging, methylation and cancer. Histol Histopathol. 2000;15:83542.

116. Matsubayashi H, Sato N, Brune K, et al. Age- and disease-related methylation of multiple genes in nonneoplastic duodenum and in duodenal juice. Clin Cancer Res 2005;11:573-83.

117. Matsubayashi H, Sato N, Fukushima N, et al. Methylation of cyclin D2 is observed frequently in pancreatic cancer but is also an age-related phenomenon in gastrointestinal tissues. Clin Cancer Res 2003;9:1446-52.

118. Waki T, Tamura G, Sato M, et al. Age-related methylation of tumor suppressor and tumor-related genes: an analysis of autopsy samples. Oncogene 2003;22: 4128-33.

119. Issa JP, Ottaviano YL, Celano P, et al. Methylation of the oestrogen receptor CpG island links ageing and neoplasia in human colon. Nature Genet 1994;7:53640.

120. Issa JPJ, Vertino PM, Boehm CD, et al. Switch from mono-allelic to bi-allelic human IGF2 promoter methylation during aging and carcinogenesis. Proc Natl Acad Sci USA 1996;93:11757-62.

121. Ahuja N, Li Q, Mohan AL, et al. Aging and DNA methylation in colorectal mucosa and cancer, Cancer Res 1998;58:5489-94.

122. Schuebel KE, Chen W, Cope L et al. Comparing the DNA hypermethylome with gene mutations in human colorectal cancer. PLoS Genet 2007;3:1709-23.

123. Knudson AG Jr. Mutation and cancer: statistical study of retinoblastoma. Proc Natl Acad Sci USA 1971;68:820-3.

124. Mc Garvey KM, Van Nestle L, Cope L, et al. Defining a chromatin status that characterizes DNA-hypermethylated genes in colon cancer cells. Cancer Res 2008;68: 5753-59.

125. Takeshima H, Yamashita S, Shimazu T, et al. Effects of genome architecture and epigenetic factors on susceptibility of promoter CpG islands to aberrant DNA methylation induction. Genomics 2011; 98:182-8.

126. Tiwari VK, McGarvey KM, Licchesi JD,
PcG proteins, DNA methylation, and gene repression by chromatin looping. PloS Biol 2008; 6:2911-27.

127. Henikoff S. Nucleosome destabilization in the epigenetic regulation of gene expression. Nat Rev Genet 2008;9:15-26.

128. Lin JC,Jeong S, Liang G, et al. Role of nucleosomal occupancy in the epigenetic silencing of h MLH1 CpG Island. Cancer Cell 2007;432-44.

129. Frigola J, Song J, Stirzaker C, et al. Epigenetic remodeling in colorectal cancer results in coordinate gene suppression across an entire chromosome band. Nat Genet. 2006;38:540-9.

130. Hitchins MP, Lin VA, Buckle A, et al. Epigenetic inactivation of a cluster of genes flanking MLH1 in microsatelliteunstable colorectal cancer. Cancer Res 2007;67:9107-116.

131. Weisenberger DJ, Siegmund KD, Campan $\mathrm{M}$, et al. CpG island methylator phenotype underlies sporadic micro-satellite instability and is tightly associated with BRAF mutation in colorectal cancer. Nat Genet 2006;38:787-93.

132. Epstein CJ, Martin GM, Schultz AL, Motulsky AG. Werner's syndrome a review of its symptomatology, natural history, pathologic features, genetics and relationship to the natural aging process. Medicine (Baltimore) 1966;45:177-221.

133. Leistritz DF, Hanson NB, Martin GM, Oshima J. Werner Syndrome. Gene Reviews 2007. Available from: www.ncbi. nlm.nih.gov/books/

134. Huang S, Lee L, Hanson NB, et al. The spectrum of WRN mutations in Werner syndrome patients. Hum Mutat 2006;27: $558-67$.

135. Goto M, Miller RW, Ishikawa Y, Sugano H. Excess of rare cancers in Werner syndrome (adult progeria). Cancer Epidemiol Biomarkers Prev1996;5:239-46.

136. Yamamoto K, Imakiire A, Miyagawa NA, et al. Report of two cases of Werner's syndrome and review of the literature $\mathrm{J}$ Orthop Surg (Hong Kong) 2003;11:22433.

137. Salk D, Au K, Hoehn H, et al. Cytogenetics of Werner's syndrome cultured skin fibroblasts: variegated translocation mosaicism Cytogenet Cell Genet 1981; 30:92-107.

138. Epstein CJ, Motulsky AG. Werner syndrome: entering the helicase era. Bioessays1996;18:1025-7.

139. Bachrati CZ, Hickson ID. RecQ helicases: suppressors of tumorigenesis and premature aging. journal Biochem J 2003;15: 577-606.

140. Rossi ML, Ghosh AK, Bohr VA. Roles of Werner syndrome protein in protection of genome integrity. DNA Repair 2010;9:331-
44.

141. Christensen BC, Houseman EA, Marsit CJ, Aging and Environmental Exposures Alter Tissue-Specific DNA Methylation Dependent upon CpG Island Context. Plos Genet 2009:5:e1000602.

142. Hutchinson CJ, Worman HJ. A-type lamins: guardians of the soma? Nat Cell Biol 2004;6:1062-7.

143. Broers JL. Ramaekers FC, Bonne G, et al. Nuclear lamins: laminopathies and their role in premature ageing. Physiol Rev 2006;86:967-1008.

144. Stadelmann B, Khandjian E, Hirt A, et al. Repression of nuclear lamin $A$ and $C$ gene expression in human acute lymphoblastic leukemia and non-Hodgkin's lymphoma cells. Leuk Res 1990;14;815-82.

145. Johnson BR, Nitta RT, Frock RL, et al. Atype lamins regulate retinoblastoma protein function by promoting subnuclear localization and preventing proteasomal degradation. Proc Natl Acad Sci USA 2004; 101:9677-82.

146. Addo BK, Chung W, Shen L, et al. AgeRelated DNA Methylation Changes in Normal Human Prostate Tissues. Clin Cancer Res 2007;13:3796-802.

147. Maegawa S, Hinkal G, Kim HS, et al. Widespread and tissue specific age-related DNA methylation changes in mice. Genome Res 2010; 20:332-40.

148. Takasugi M. Progressive age-dependent DNA methylation changes start before adulthood in mouse tissues. Mech Ageing Dev 2011;132:65-71.

149. Thompson RF, Atzmon G, Gheorghe C, et al. Tissue-specific dysregulation of DNA methylation in aging. Aging Cell 2010;9: 506-518

150. Wagner W, Bork S, Horn P, et al. Aging and Replicative Senescence Have Related Effects on Human Stem and Progenitor Cells. PLoS ONE 2009;4: e5846.

151. Teschendorff AE, Menon U, GentryMaharaj A, et al. Age-dependent DNA methylation of genes that are suppressed in stem cells is a hallmark of cancer. Genome Res 2010;20:440-6

152. Koch CM, Suschek CV, Lin Q, et al. Specific age-associated DNA methylation changes in human dermal fibroblasto. PLoS One 2011;6:e16679.

153. Bork S, Pfister S, Witt H. DNA methylation pattern changes upon long-term culture and aging of human mesenchymal stromal cells. Aging Cell 2010;9:54-63.

154. Mehta IS, Figgitt M, Clements CS, et al. Alterations to Nuclear Architecture and Genome Behavior in Senescent Cells. Ann N Y Acad Sci 2007;1100:250-63. 